\title{
Trust Model Research in Cloud Computing Environment
}

\author{
Haiyang Ding ${ }^{\mathrm{a}}$, Xiguang Li ${ }^{\mathrm{b}}$, Changqing Gong ${ }^{\mathrm{c}}$ \\ Computer department, Shenyang Aerospace University, Shenyang, \\ 110136, China \\ Jocean.cool@163.com, ${ }^{b}$ lixiguang@sau.edu.cn, ${ }^{c}$ gongchangqing@sau.e \\ du.cn
}

\begin{abstract}
.
The trust evaluation research in cloud computing has been involved in the node security communication, security storage, resource allocation and many other aspects.As an effective replacement oftraditional networksecurity,trust mechanismhas effectively solved the security problems of distributed computing, such as grid computing, pervasive computing and adhoc networks. The classical trust model and research status of trust model in cloud computing have been studied.Considering the factors such as time, a trust model based on Evidence Theory has been proposed in this paper. This model limits the qualification of the entity's recommendation trust, proposes a dynamic allocation method of trust weights and gives thecalculation method of trust value.
\end{abstract}

Keywords: cloud computing, trust model, Evidence Theory

\section{Introduction}

Cloud computing is a kind of information service mode based on grid computing, it has the advantages of large scale, high reliability and extremely cheap, so it is widely used. Cloud computing distribute computing task to the resource pool, which constitutedby a large number of computers [1].Users can obtain the service of computing, storage space and application according to their needs. Cloud computing is a kind of computingservicemode, by which the 
computing resourcescan be acquiredeasily, efficiently, according to demand $[2,3]$.These resources can be quickly supply and release, only need a minimum of the interaction between the user and service providers.

At present, there are a lot of public cloud service providers, such as Google's GFS (Google File System), Amazon’s E2C, Microsoft's Windows Azure, etc. Cloud computing provides a "pay as you use" service mode, users use the cloud resources and services through the way of paying. With the popularization and development of cloud computing, users will face a more and more important problem: how to choose a most trusted service from a lot of cloud services, to prevent the cloud service provider offers shoddy service,to obtain satisfactory service to the greatest extent $[4,5]$. In order to solve this problem, we have to evaluate the credibility of the service quality in cloud computing environment. So it is necessary to consider the trust issues in the process of selecting the cloud service and consider the trust problem within each entity in cloud computing.

\section{Research status}

Through analyzinga lot of trust model references, according to different trust value representation methods, the trust model can be divided into the following four types.

(1) A trust model based on probability [6]. This trust model uses the method of probability to describe the trust value, the trust is divided into direct trust and indirect trust at the same time.According to certain experience and negative experience, the probability of entity to complete the task can be computed.This probability can be regarded as a measure of entitytrust,the trust comprehensive calculation formula can be derived also.

(2) A trust model based on fuzzy theory [7]. This trust model usesfuzzy theory to discussthe credibility of the entity, using the concept of characteristic vector and membership degree, realizingthe quantitative description of trust. Based on fuzzy theory, this trust calculation method canavoid the either/or exclusive relationship, solve the problems of the fuzziness of trust.

(3) A trust model based on cloud theory [8]. This model uses trust cloud to 
express trust relationship. The trust cloud is a special kind of cloud model, it characterized by a triad (Ex, En, He) according to the trust relationship and the characteristic of description method, Ex represents the basic trust value, En represents the uncertainty of Ex, He represents the uncertainty of En.

(4) A trust model based on evidence theory $[9,10]$. This trust model divide trust into "trusted", "untrusted" and "uncertainty", using basic trust function of the evidence theory to express the three degree of trust. This trust modelcan express the uncertainty trustrelationeffectively,otherwise, the probabilitytrust model can only express the binary trustrelation.

\section{Trust model based on Evidence Theory}

In cloud computing environment,there are varies trustrelationbetween entities. These entities include network entity, storage entity and computing entity, distributing in cloud users and cloud servers. This trustrelationship can be established through the center of cloud resource management, and the trust degreeof each entity can be verified in interactingprocess. In view of the existence of a large number of uncertainty factors in a cloud environment, the trust relationship should bedivided into three types: entity trusted (TR), entity untrusted (NT) and uncertainty trusted (UN), the trust relationshipcan be expressed by (TR, NT, (TR,NT)). Trust model based on Evidence Theoryis shown in Figure1. 


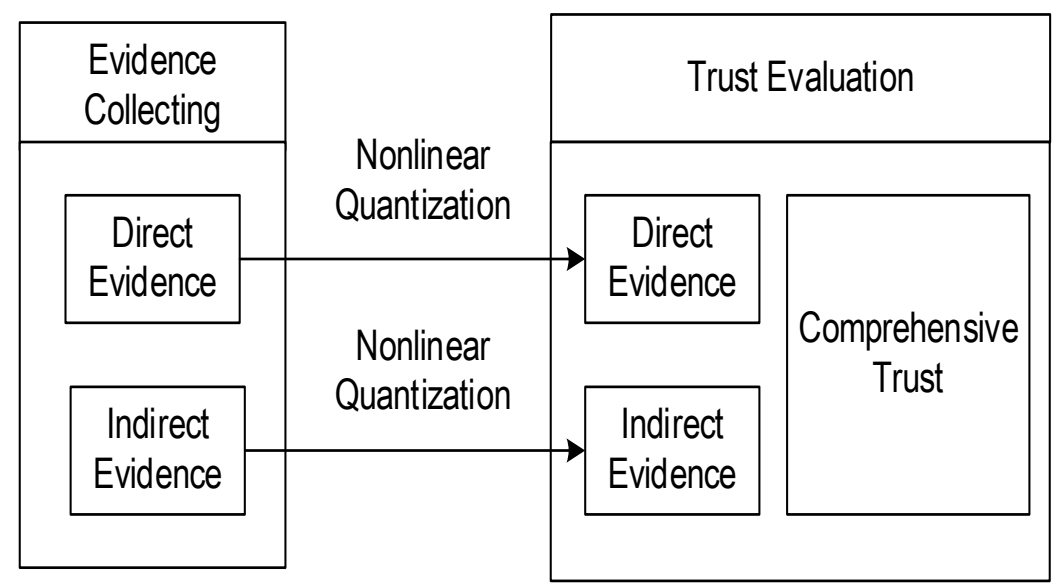

Figure.1 Trust model based on Evidence Theory

The process of trust decision is decided by the comprehensive trust value which isa combination of direct trust value and indirect trust value [11].

\section{Direct trust value}

Direct trust value is expressed with TD.Thecloud center set a time period $T_{c}$, because trust will decay over time, so we only consider the condition of entities directly interact within the time interval $\left[t-T_{c}, t\right]$. In this period, the direct trust value of entity $i$ to entity $j$ is $\mathrm{TD}_{\mathrm{i}, \mathrm{j}}$, the formulais as follows.

$$
\left\{\mathrm{m}(\mathrm{TR})=\left\{\begin{array}{cc}
\mathrm{TD}_{\mathrm{i}, \mathrm{j}}=(\mathrm{m}(\mathrm{TR}), \mathrm{m}(\mathrm{NT}), \mathrm{m}(\mathrm{TR}, \mathrm{NT})) \\
\frac{\mathrm{a}_{\mathrm{i} 1}^{2} \times 0.618}{\mathrm{a}_{\mathrm{i} 1}^{2}+\mathrm{a}_{\mathrm{i} 2}^{2}+\mathrm{a}_{\mathrm{i} 3}^{2}}, & \text { if } \mathrm{a}_{\mathrm{i} 1}+\mathrm{a}_{\mathrm{i} 2}+\mathrm{a}_{\mathrm{i} 3} \leq 100 \\
0.618+(1-0.618) \times \frac{\log _{10} \mathrm{a}_{\mathrm{i} 1}}{\log _{10} \mathrm{a}_{\mathrm{i} 1}+\log _{10} \mathrm{a}_{\mathrm{i} 2}+\log _{10} \mathrm{a}_{\mathrm{i} 3}}, & \text { else } \\
\mathrm{m}(\mathrm{NT})=\left\{\begin{array}{cc}
\frac{\mathrm{a}_{\mathrm{i} 2}^{2} \times 0.618}{\mathrm{a}_{\mathrm{i} 1}^{2}+\mathrm{a}_{\mathrm{i} 2}^{2}+\mathrm{a}_{\mathrm{i} 3}^{2}}, & \text { if } \mathrm{a}_{\mathrm{i} 1}+\mathrm{a}_{\mathrm{i} 2}+\mathrm{a}_{\mathrm{i} 3} \leq 100 \\
0.618+(1-0.618) \times \frac{\log _{10} \mathrm{a}_{\mathrm{i} 2}}{\log _{10} \mathrm{a}_{\mathrm{i} 1}+\log _{10} \mathrm{a}_{\mathrm{i} 2}+\log _{10} \mathrm{a}_{\mathrm{i} 3}}, & \text { else }
\end{array}\right. \\
\mathrm{m}(\mathrm{TR}, \mathrm{NT})=1-\mathrm{m}(\mathrm{TR})-\mathrm{m}(\mathrm{NT})
\end{array}\right.\right.
$$

$\mathrm{a}_{\mathrm{i} 1}, \mathrm{a}_{\mathrm{i} 2}, \mathrm{a}_{\mathrm{i} 3}$ are the trusted number, untrusted number and uncertainty number 
respectively of the interval between entity $i$ and entity $j$. If entity $i$ and entity $j$ have no interaction occurred in the time interval $\left[t-T_{c}, t\right]$, set $T_{i, j}$ to $(0,0$, 1),that is the maximum of uncertain degree.

\section{Indirect trust value}

Indirect trust valueisexpressed by IND. For acquiring indirect trust value of entity $i$ to entity j,it is need the recommendation of other entities who ever had interaction with entity $\mathrm{j}$, recommendation trust value is represented by REC. The recommendation trust value of entity $k$ to entity $\mathrm{j}$ is as follows.

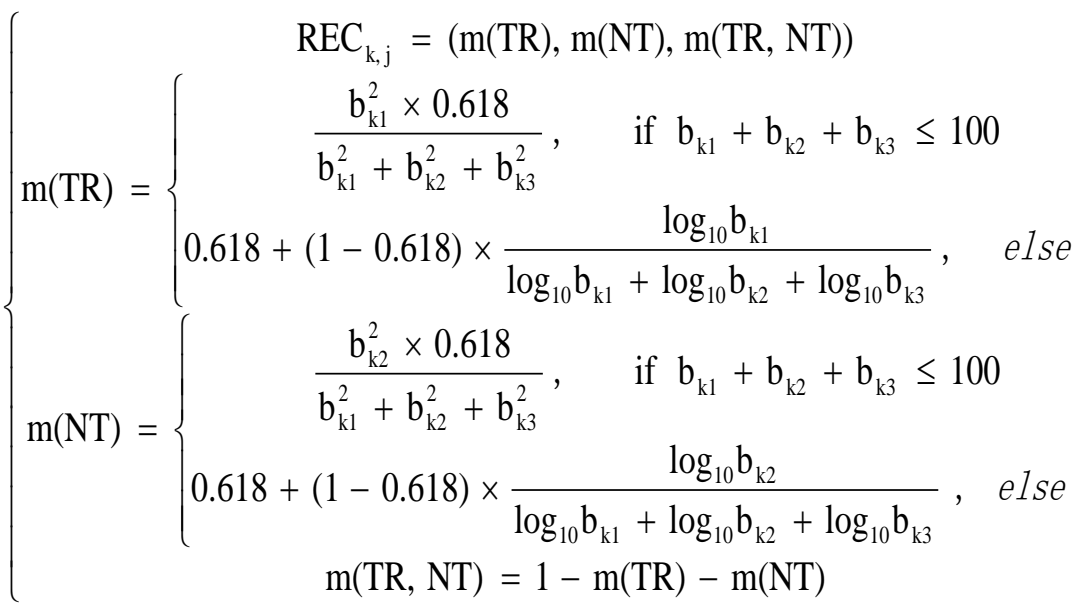

$b_{k 1}, b_{k 2}, b_{k 3}$ are the trusted number, untrusted number and uncertainty number respectively of the interval $\left[t-T_{c}, t\right]$ between the recommend entity $k$ and entity j.

As there may be multiple recommendation entities, but not all of these entities can be trusted. Using the unreliable entity's recommendation trust value will eventually affect the accuracy of the trust value. Sowe need to set athresholdfor all the recommend entities, herewe set the threshold value not less than 0.5 , only suchentity have the recommended qualification.If the number of recommend entity is $n$, indirect trust value is obtained as follows [12].

$$
\mathrm{IND}_{\mathrm{i}, \mathrm{j}}=\mathrm{REC}_{1, \mathrm{j}} \otimes \mathrm{REC}_{2, \mathrm{j}} \otimes \cdots \otimes \mathrm{REC}_{\mathrm{n}, \mathrm{j}}
$$

\section{Comprehensive trust value}


The comprehensive trust value of entity $i$ to entity $\mathrm{j}$ is as follows.

$$
\mathrm{T}_{\mathrm{i}, \mathrm{j}}=\alpha \mathrm{TD}_{\mathrm{i}, \mathrm{j}}+\beta \mathrm{IND}_{\mathrm{i}, \mathrm{j}}
$$

Factor $\alpha$ is direct trust weight, $\beta$ is indirect trust weight, and $\alpha+\beta=1$. In time interval $\left[t-T_{c}, t\right]$, if the direct interaction times is $m$ between entity $i$ and entity $j$, and the number of entity who have recommendation qualification is $\mathrm{n}$ in indirect trust,the value of $\alpha$ and $\beta$ are as follows.

$$
\left\{\begin{array}{l}
\alpha=\frac{m}{m+n} \\
\beta=\frac{n}{m+n}
\end{array}\right.
$$

If the interaction timesbetween entity $i$ and entity $j$ is a large number, then $\mathrm{TD}_{\mathrm{i}, j}$ will get a bigger weight. If the number of entity who have recommendation qualification is large enough, then $\operatorname{IND}_{\mathrm{i}, \mathrm{j}}$ will get a bigger weight. This kind of dynamic weighting factor strategy can evaluate the entity's trustmore accurately.

\section{Performance analysis}

For initial state, due to the interaction times between entity $i$ and entity jis few, and the entities who have recommended qualification is also few, so $T_{i, j}$ will have a lower trust degree, its uncertain degree is higher. As the increasing of interaction times between each entities, the interaction times of entity $i$ and entity $\mathrm{j}$ will be enhanced and the entities who have recommended qualification will be enhancedalso, then the trust degree of $T_{i, j}$ will increase and the uncertainty degree will decrease. When the interaction times is enough between each entity, the trust degree of $T_{i, j}$ tends to be stable, the degree of uncertainty is reduced to some extent and then tend to be smooth.

\section{Conclusion}

Considering the factors such as trust time, basing on evidence theory, a trust value calculation method has been put forward in this paper.It limits the recommended qualification ofentities, inorder to avoid malicious evaluation from 
low trust entity.Our trust model also adopt dynamic weight distribution to makemore accurate assessment of the entity's trust.

\section{Acknowledgements}

This research is supported by Education Department of Liaoning Province, the foundation No. is L2013064. This research is supported by Technology Innovation Foundation (Basic Research) of Aviation Industry Corporation of China, the foundation No. is 2013S60109R. This research is supported by Natural Science Foundation of Liaoning Province, the foundation No. is 201102171.

\section{References}

[1] Dengguo Feng, Min Zhang, Yan Zhang, Zhen Xu: Journal of Software Vol. 22 (2011), p. 71

[2] Xiaoyong Li, Xiaolin Gui: Journal of Software Vol. 6 (2007), p. 1510

[3] Wang M C, Wu X, Zhang W: Proceeding of 2011 IEEE 9th International Conference on Dependable Autonomic and Secure Computing (2011)

[4] Xuan Wang, Lei Wang: Intelligent Systems Design and Applications Vol. 2 (2008), p. 591

[5] Jianli Hu, Quanyuan Wu: Journal of Computer Science Vol. 9 (2009), p. 1

[6] Ping Wang, Jing Qiu: Fuzzy Systems and Knowledge Discovery (2011)

[7] Schmidt S, Steele R, Dillon TS: Applied Soft Computing Journal Vol. 7 (2007), p. 492

[8] Zhaoxiong Zhou, He Xu, Suoping Wang: Advances in Information Sciences and Services Sciences (2011)

[9] Kai Wei, Shaohua Tang: Computational Intelligence and Security Vol. 2 (2009), p. 411 
[10] Yanchun Zhu, Wei Zhang: IT in Medicine and Education (2008)

[11] Enguang Fang: Trust model research based on behavior results in cloud computing environment (Hangzhou Dianzi University, China 2011)

[12] Xiaonian Wu, Runlian Zhang: Procedia Computer Science (2013), p. 1170 